\title{
Fabricación, desarrollo y programación de un rover agricultor tele operado con RF y monitoreado mediante sistema SCADA.
}

\section{Manufacture, development and programming of a RF- operated and SCADA-monitored farmer rover.}

Nohelia Cruz, Std ${ }^{1}$, Leonardo Enamorado, $\mathrm{Std}^{2}$, Luis Romero, Std ${ }^{3}$, Héctor Santos, $\mathrm{Std}^{4}$, Allan Castellanos,Std ${ }^{1}$ Universidad Tecnológica Centroamericana, Honduras, nohelia19999@unitec.edu, ${ }^{2}$ Universidad Tecnológica Centroamericana, Honduras,leoenamorado@unitec.edu, ${ }^{3}$ Universidad Tecnológica Centroamericana, Honduras, ferromero@ unitec.edu, ${ }^{4}$ Universidad Tecnológica Centroamericana, Honduras, hectorjsp18@gmail.com, ${ }^{5}$ Universidad Tecnológica Centroamericana, Honduras, allan.castellanos94@gmail.com, 


\title{
Fabricación, desarrollo y programación de un rover agricultor tele operado con RF y monitoreado mediante sistema SCADA.
}

\section{Manufacture, development and programming of a RF- operated and SCADA-monitored farmer rover.}

\author{
Nohelia Cruz, Std ${ }^{1}$, Leonardo Enamorado, $\mathrm{Std}^{2}$, Luis Romero, Std ${ }^{3}$, Héctor Santos, $\mathrm{Std}^{4}$, Allan Castellanos,Std ${ }^{5}$ \\ ${ }^{1}$ Universidad Tecnológica Centroamericana, Honduras, nohelia19999@ unitec.edu, ${ }^{2}$ Universidad Tecnológica Centroamericana, \\ Honduras,leoenamorado@unitec.edu, ${ }^{3}$ Universidad Tecnológica Centroamericana, Honduras, ferromero@ unitec.edu, ${ }^{4}$ Universidad \\ Tecnológica Centroamericana, Honduras, hectorjsp18@gmail.com, ${ }^{5}$ Universidad Tecnológica Centroamericana, Honduras,
} allan.castellanos94@gmail.com,

Resumen-_ El crecimiento global y el cambio climático han implicado una intensificación de la agricultura en los últimos años, La necesidad de aplicar nuevas tecnologías de automatización que garanticen el desarrollo sostenible de los procesos agrícolas paralelo a un uso razonable de los recursos naturales del medio ambiente es una prioridad para aumentar el rendimiento, la eficiencia y la calidad de los cultivos, rentabilidad de los agricultores y reducción de los costes de producción. En el presente trabajo se desarrolla la implementación de un robot tele operado por radio frecuencia para operaciones en el proceso de siembra del sector agrícola. El rover está habilitado para la obtención de datos sensoriales y transmisión de estos de forma inalámbrica por medio de radio frecuencia de 2. $4 G H z . E l$ propósito principal de la implementación de un rover tele operado es incentivar a la adopción de tecnología robótica, en pequeños y medianos agricultores en Honduras. Para la fabricación del prototipo se tomaron en cuenta las múltiples variabilidades de movilización, interacción, condiciones de siembra y riesgos que pueden ocurrir al momento de interactuar con el campo de trabajo, así como las áreas en que se puede implementar un rover. La fabricación del prototipo involucra el desarrollo electrónico, mecánico estructural y la lógica de programación de ambos microprocesadores PIC18f45k22 y Atmega328p dando como resultado un agrobot operable en terrenos llanos con un efector final capaz de perforar un agujero de $4 \mathrm{~cm}$ de profundidad y depositar un máximo de tres semillas de frijol.

Palabras clave: Robótica, microprocesadores, radio frecuencia, efector final.

Abstract- Global growth and climate change have meant intensification of agriculture in recent years, the need to apply new automation technologies to ensure the sustainable development of agricultural processes in parallel with the reasonable use of natural resources in the environment is a priority to increase yield, efficiency and quality of crops, profitability of farmers. In the present work, the implementation of a remote-operated radio frequency robot is developed for operations in the sowing process of the agricultural sector. The rover is enabled for the collection of sensory data and transmission of these wirelessly by means of radio frequency of 2. $4 \mathrm{GHz}$. The main purpose of the implementation of a remotely operated rover is to encourage the adoption of robotic technology, in small and medium farmers in Honduras. The production of the prototype considered the multiple variabilities of mobilization, interaction, seeding conditions and risks that can occur when interacting with the field of work, as well as the areas in which a rover can be deployed. Prototype manufacturing involves electronic development, structural mechanics and programming logic of both microprocessors PIC18f45k22 and Atmega328p resulting in an agrobot operable on flat terrain disposed of an end effector capable of drilling a hole 4cm deep and depositing a maximum of three bean seeds.

$$
\begin{array}{r}
\text { Keywords-Robotics, microprocessors, radio frequency, end } \\
\text { effector. }
\end{array}
$$

\section{INTRODUCCION}

Para el 2050, se estima que el crecimiento poblacional alcanzara aproximadamente 9.1 billones, lo que representa $34 \%$ más de la población actual. Para alimentar este crecimiento masivo la producción de alimentos debe incrementar en un $70 \%$ [1].

Con el continuo desarrollo de la sociedad, la ciencia y la tecnología, lo tradicional y tosco en la gestión del cultivo de plantación manual ya no se utiliza en el desarrollo de la agricultura moderna en países desarrollados. En cambio, se ha implementado un modelo de información digital para la gestión agrícola. No obstante este sector no siempre se vio favorecido ante la innovación tecnológica, la agricultura ha tenido un proceso evolutivo con el pasar de los anos viéndose notorias diferencias entre sus métodos de siembra y sus niveles de producción[2].En sus inicios la productividad era relativamente baja y controlada, ya que el ser humano usaba su fuerza para todas las labores que conlleva la agricultura, así como el uso de herramientas manuales simples (hoces y palas) y se auxiliaba de la fuerza de animales para operaciones más arduas como arar la tierra [3]. En el siglo 19 con la primera revolución industrial y el desarrollo de las maquinas a vapor. La introducción de 
maquinaria agrícola operada por el granjero manualmente y el uso de químicos fertilizantes, a pesar de que hubo un incremento considerable de la eficiencia y productividad en la agricultura con la facilidad de trabajo que ofrecían las innovaciones tecnológicas, consigo también trajo un excesivo consumo de energía por las maquinas que funcionaban a gasolina, una contaminación química en el campo de trabajo, destrucción del ambiente y desperdicio de recursos naturales [3]. En el siglo 20 la invención de las computadoras y el desarrollo de la programación facilito la apertura a la creación de mecanismos multioperacionales autómatas con la capacidad de reemplazar al ser humano en ciertas operaciones de la vida diaria. El sector agrícola no fue la excepción al verse beneficiado con el rápido desarrollo de la electrónica y computación para aplicar estas técnicas robóticas en la mejora de su maquinaria y consigo realizar operaciones de manera inteligente y eficiente. La distribución de tareas redujo el uso de químicos y mejoro la precisión en los procesos como la irrigación y la siembra [14], [15]. Hoy en día, esta nueva era de la agricultura presenta un nuevo concepto "la agricultura de precisión" cuyo objetivo es observar, medir y responder a la variabilidad del campo auxiliándose de la tecnología para el análisis de datos como el internet de las cosas, la inteligencia artificial, Big Data, Cloud Computing y el control de sensores remotos entre otros. [13], [16]

Existe un contraste entre el sector agrícola de países desarrollado y el de América Latina, hasta el día el de hoy las actividades agrícolas en Latinoamérica siguen siendo primordialmente dominadas por tecnologías obsoletas y arcaicas, utilizando técnicas que datan de periodos de la colonización y que repercute en las prácticas ineficientes de los pequeños agricultores y en su deterioro físico [1]. Estudios demuestran que la prevalencia de dolor cervical por la producción de tabaco en el sur de Brasil fue del 33\% de mujeres ordeñadoras y el $26 \%$ de trabajadores rurales, entre la población estudiada. Esto se da debido a que existen malas condiciones laborales, y los trabajos y movimientos repetitivos que se llevan a cabo en su área de trabajo, dan paso para el desgaste muscular y fisiológico de la persona [2]. Los productores pequeños que no forman parte de un conglomerado mayor suelen desarrollar los procesos de siembra de manera empírica, desconociendo técnicas de cultivos que maximicen la productividad y minoricen gastos y tiempo laboral. Se ignoran variables del campo que afectan el rendimiento de las cosechas como los arreglos espaciales y distribución de las semillas, las profundidades de siembra y el uso correcto del agua [3]. En Honduras, la mayor parte de la producción se recoge manualmente. Esta recolección manual conduce a un flujo de trabajo lento durante los días más intensos de la cosecha. Viene en el coste de horas adicionales de trabajo, incomodidad del cliente, y por encima de eso, todos los datos se almacenan en el papel[7].

La necesidad conduce a la implementación de tecnologías en el sector agrícola en la dirección del uso de microcontroladores, maquinas o sistemas de planeación y manejo. Actuadores mecánicos son dispositivos usados en el desarrollo de mecanismos construidos como ayuda en los procesos agrícolas, agro robots o maquinas que plantan, recolectan, aran o regeneran la tierra. Los sensores por su parte son vitales para la agricultura digitalizada e inteligente, recolectando, almacenando y procesando todo tipo de información censada sobre los aspectos ambientales que afectan el entorno del cultivo [8]. Al implementar la robótica en la agricultura, se aumenta la productividad, la eficiencia, la precisión y la confiabilidad en el área de cosecha y plantación. Los ambientes complejos y con variabilidad en luminosidad, temperatura y humedad en ambientes de agricultura, requieren de sistemas robustos para apoyar labores agrícolas de forma segura [5].

La tele operación de un robot, por su parte permite realizar diferentes tareas en entornos remotos. Los sistemas de tele operación se componen de un sitio local y un sitio remoto. El sitio local donde un operador maneja un manipulador llamado maestro. El sitio remoto, donde un manipulador llamado esclavo, interactúa con el mundo físico y un canal de comunicación que enlaza ambos sitios[9].

Aunque los robots móviles reducen las necesidades de mano de obra humana, los robots no sustituirán por completo a los trabajadores, pero ciertamente les permitirán centrarse en otras tareas distintas del transporte de mercancías [10]. Dicho esto, la presente investigación se hace con el objetivo de mejorar los procesos de siembra, reduciendo los tiempos de trabajo; asimismo se prevé un panorama más amplio y claro de la rentabilidad de los dispositivos robóticos en el sector agrícola para los agricultores en proceso de adopción tecnológica. Este estudio conduce al desarrollo y fabricación de un rover tele operado por radio frecuencia, habilitado para la obtención de datos sensoriales y transmisión de estos de forma inalámbrica por medio de radio frecuencia de $2.4 \mathrm{GHz}$. Su función es logra agilizar el proceso de siembra en los campos agrícolas siendo capaz de sembrar semillas con la ayuda de un efector final de siembra capaz de perforar agujeros con profundidades de $4 \mathrm{~cm}$ y de dispensar semillas.

\section{MARCO TEÓRICO}

Para el desarrollo de sistemas mecatrónicos, existe un modelo más flexible a seguir, este es basado en tres elementos que son: el modelo en forma de $\mathrm{V}$ en un nivel macro, ciclo general de resolución de problemas en el micro nivel y por último módulos de procesos predefinidos para repetir los pasos de la operación durante el diseño de los sistemas mecatrónicos.

\section{A. Modelo en $\mathrm{V}$}

El modelo en forma de $\mathrm{V}$ esta establecido en el dominio de la ingeniería de software y partiendo de ahí se ha adaptado a las necesidades de la mecatrónica. Esta metodología describe el procedimiento genérico para el diseño de sistemas mecatrónicos que deben especificarse de acuerdo con la tarea de diseño individual.

\section{B. Software}

Para la implementación de un robot en cualquier aspecto en el que este se encuentre, la programación de sus funciones es 
simplemente vital para el desarrollo correcto de sus tareas. La integración correcta de las ordenes programadas dentro del procesador, y la comunicación con el mundo exterior por medio de sensores o señales obtenidas, son las bases para el funcionamiento adecuado de cualquier sistema "inteligente". Existe una amplia variedad de lenguajes de programación, softwares de compilación, así como diferentes softwares para diseñar un sistema para el monitoreo continuo de aplicaciones robóticas, además de múltiples librerías que en ellas integran el desglose de señales análogas o digitales que se pueden recibir mediante sensores. En la investigación actual para la programación y diseño electrónico de un robot agrónomo, se implementaron diferentes softwares como MikroC, el entorno de desarrollo integrado de Arduino, Microsoft Visual Studio, entre otros, para el desarrollo de prototipaje correcto de los componentes por utilizar.[6]

\section{Microprocesador}

A veces denominados controlador integrado o unidad de microcontrolador (MCU), los microcontroladores se encuentran en robots, computadoras, dispositivos médicos, transceptores, máquinas industriales y electrodomésticos, entre otros dispositivos. Son esencialmente computadoras miniatura simples diseñadas para controlar pequeñas funciones de un componente más grande, sin un complejo de interfaz de usuario. Un microcontrolador está integrado dentro de un sistema para cumplir una función en un dispositivo, interpretando los datos que recibe de sus periféricos de entrada y salida utilizando su procesador central.

\section{Efector final de siembra}

Un robot agricultor está conformado por tres estructuras principales: el equipo de locomoción, la estructura manipuladora y el efector final. Cada uno cumple una función importante para lograr el objetivo final, el equipo de locomoción se encarga de trasladar la estructura manipuladora y al efector final a su área de destino. La diferencia entre un robot agricultor y otro se aprecia según el tipo de subsistema mecatrónico de pinza (efector final) que tenga; para sembrar, cosechar, quitar maleza etc,.La mayoría de las plataformas robóticas son sobre ruedas [11] y en términos de la estructura del manipulador, la solución elegida es un mecanismo suspendido o un brazo fijo sobre el cual se monta los componentes del sistema de perforación: el pistón, el husillo motorizado y la herramienta de perforación. El desplazamiento en el eje vertical controla la profundidad del surco en la tierra y el diámetro de la herramienta determina así mismo el diámetro del agujero.

E. Sistema de tasa de siembra variable

Los últimos modelos de sistemas de accionamiento de dosificadores han migrado de accionamientos hidráulicos a motores eléctricos, estos últimos suelen ser menos costosos, pero los accionamientos electrónicos suelen girar a menores velocidades debido al accionamiento directo de naturaleza del mecanismo. Las sembradoras de accionamiento eléctrico suelen utilizar motores paso a paso o un motor de $\mathrm{CC}$ con reducción de engranajes. Con esta independencia entre el mecanismo transportador y el dispensador de semilla se logra que el control de la tasa de dispersión de semillas varíe según las exigencias del usuario y no con respecto a la velocidad de la estructura que lo traslada[5]. Mediante medios electrónicos, comandos de operación asignados en un microcontrolador, datos sensoriales y dispositivos mecánicos se controla la tasa de dispersión de un dispensador de semillas en tiempos o momentos específicos dictados por el usuario y que no dependen necesariamente del artefacto móvil sobre el cual va montado.

Con la incorporación de componentes electrónicos en el diseño del efector, se pueden sustituir los componentes meramente mecánicos y reducir el tamaño de los mecanismos utilizados para la siembra, formando un diseño más compacto que utilice actuadores eléctricos lineales y giratorios para la trasmisión de movimientos.

\section{METODOLOGÍA}

Se utilizo la metodología en "V" (Figura1), siendo la más adecuada para la fabricación de productos mecatrónicos, orientada al campo de la robótica. El modelo consiste en dos ciclos de dos etapas obteniendo un producto funcional al completar el segundo ciclo.

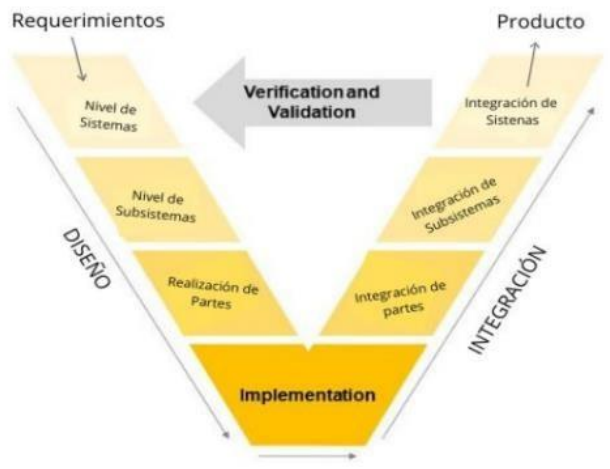

Figura. 1. Metodología en "V".

Los resultados a evaluar en este proyecto son cuantitativos ya que con estos se pueden evaluar las características del robot. Durante la realización de este proyecto las variables a considerar y evaluar son: Eficiencia energética como variable independiente y las variables dependientes que son, precisión de medición, velocidad de motores, longitud de respuesta.

\section{DESARROLLO}

Lo primero que se debe hacer en la metodología antes de comenzar con las etapas, es definir los requerimientos del proyecto. Uno de los principales requerimientos fue que los elementos y componentes a utilizar sean fáciles de adquirir para beneficio de los pequeños y medianos agricultores dentro de Honduras. Luego de identificar las necesidades del consumidor, se realiza un análisis en el cual se definen las funciones del sistema en general. En este análisis se definen dos tipos de funciones diferentes: Como función principal esta que debe satisfacer las necesidades del usuario que utilizara el producto 
en este caso el ROVER debe de ser capaz de sembrar semillas por medio de un efector final y su estructura debe admitir la locomoción para conducirse hasta su destino final. Como segunda función esta la "Restricción" en la cual se verifica que el sistema de control funcione de forma correcta y optima en el ROVER, de esta forma poder garantizar confianza y seguridad al usurario a la hora de manipular las acciones del robot de manera remota.

\section{A. Etapa 1 - Nivel de los sistemas - Ciclo:}

Siguiendo los pasos de la metodología el primer paso a seguir para desarrollar un producto mecatrónico es definir los sistemas del proyecto, se definieron 5 sistemas:

- Sistema mecánico, este sistema se compone de motores, estructura y efector final de siembra.

- Sistema de Energía, el cual se compone de baterías recargables y reguladores de voltaje para alimentar los componentes y motores del ROVER.

- Sistema de comunicaciones, en este se encuentran los microprocesadores que leen los datos detectados por los sensores.

- Sistema de Geolocalización, este sistema cuenta con un módulo GPS para poder localizar el ROVER dentro del campo de plantación.

- Sistema de Sensores y Control, aquí es donde se encuentran todos los sensores útiles para extraer datos del ambiente al usuario y asistir en la toma de decisiones en el campo de plantación.

\section{B. Etapa II: Nivel de los subsistemas}

Después de definir cada sistema se continua a determinar los subsistemas.

Subsistema Mecánico:

1. Piezas de Soporte, son las que ayudaran a mantener los motores en su lugar y el efector final acoplado a la estructura del rover.

2. Compartimiento de almacenamiento de componentes electrónicos.

3. Mecanismo de perforación para excavar el agujero de las semillas. Se utilizará un actuador lineal para extender la herramienta perforadora y un motor dc para lograr que la herramienta rote. Accionando simultáneamente ambos actuadores para una perforación eficaz de la herramienta.

4. Mecanismo dispensador de semillas para depositar las semillas una vez la operación de perforación haya terminado. Se utilizará un motor stepper para girar el rodillo a través del cual las semillas caerán por gravedad hasta el agujero.

Subsistema de Energía:

1. 4 reguladores de Voltaje, estos se encargarán de entregar el voltaje correcto a cada componente para que pueda funcionar correctamente.

2. 1 batería de litio recargable, esta se encarga de alimentar todos los componentes y motores, junto con el regulador de voltaje envían el voltaje necesario para que funcionen correctamente.

Subsistema de Comunicaciones:

1. Dos microcontroladores que procesarán la información que el sistema de control estará emitiendo, procesan y envían los datos captados por los sensores.

2. Una placa electrónica donde se montará el microcontrolador y los sensores.

3. Módulo de radiofrecuencia $2.4 \mathrm{GHz}$, por medio de este se logra la tele operación del robot y el control de sus componentes sensoriales, movimientos y accionamiento del efector final.

4. Se ocupa una estación de monitoreo con un SCADA para que el usuario pueda observar y guardar los datos obtenidos por los sensores.

Subsistema de Geolocalización:

1. Módulo de GPS, por medio de este módulo se logra localizar el robot en el terreno de plantación y así lograr un mejor distanciamiento entre semillas.

Subsistema de Sensores y Control:

En la (figura2) se muestra el sistema completo del funcionamiento del rover descompuesto en los subsistemas que logran la locomoción, la adquisición de datos y su control remoto.

1. Un higrómetro para medir la humedad del suelo donde se sembrarán las semillas y darle a conocer al usuario si el terreno es óptimo para la siembra de las semillas.

2. Sensores que midan Temperatura, humedad y presión en el aire, al igual que el higrómetro estas tres variables ayudan al usuario para saber si las condiciones son óptimas para el sembrado de las semillas.

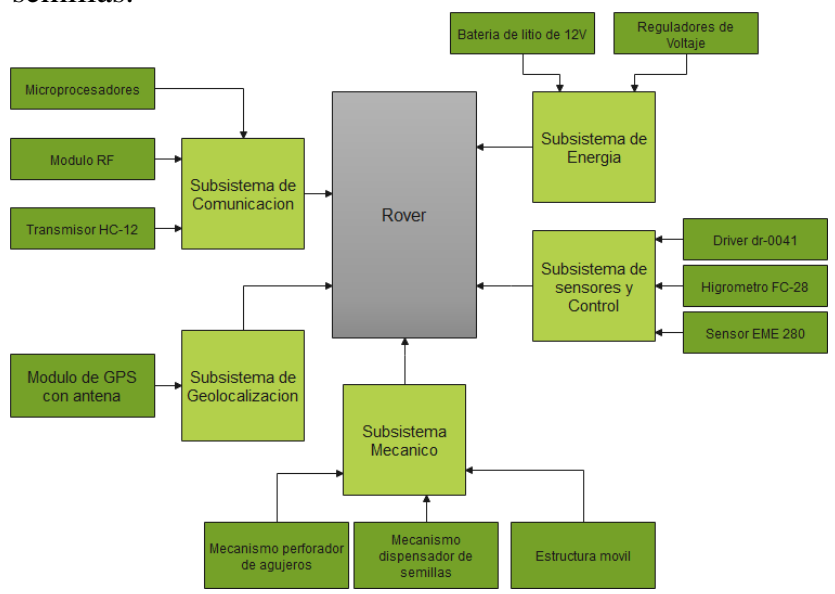

Figura. 2 Sistemas y Subsistemas del ROVER.

\section{Etapa III: Realización de Partes}

Seguidamente se eligieron los componentes específicos a utilizar en la elaboración del prototipo.

Partes Mecánicas:

- Llantas: se diseñaron las llantas en SolidWorks (figura3) y se fabricaron con manufactura aditiva con 
material TPU ya que este es un material más flexible para el terreno en el que se utilizaran.

- Armazón: se diseñó un armazón en SolidWorks con la herramienta de chapa metálica para una fabricación más fácil del mismo.
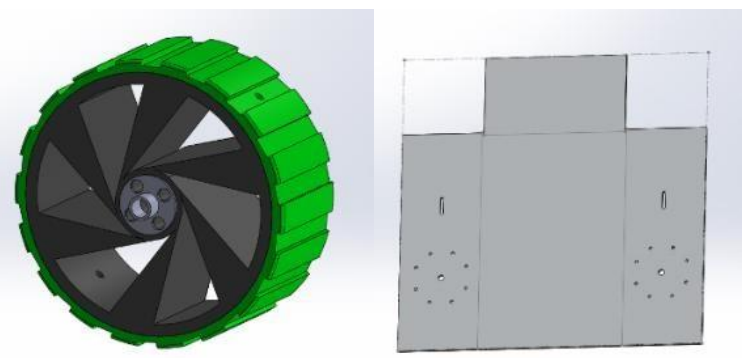

Figura. 3 Diseño de llanta y armazón.

Efector final: siguiendo con los diseños mecánicos en SolidWorks, se procedió a diseñar la herramienta perforadora y el dispensador de semillas (figura4) los cuales se colocarán en la parte frontal del robot. El dispensador de semillas se compone la tolva, rodillo, ducto dispensador y la carcasa de protección. La herramienta de perforación consta de una broca con aspas y un husillo motorizado. Se utiliza un motor stepper dc para el giro el rodillo y un motor dc para el movimiento de la broca, asimismo un actuador lineal para desplazar la herramienta.

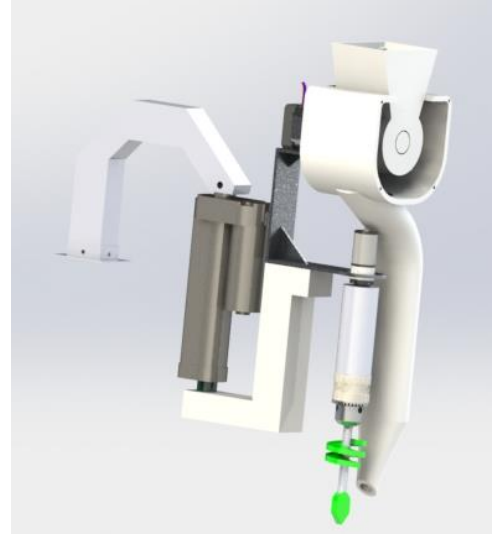

Figura. 4 Diseño de efector final.

En la (figura5) se observa el diseño de la herramienta de perforación, el husillo motorizado es un elemento adquirido del comercio en cambio los soportes y la broca se fabricaron de aluminio y de acero HSS, respectivamente.

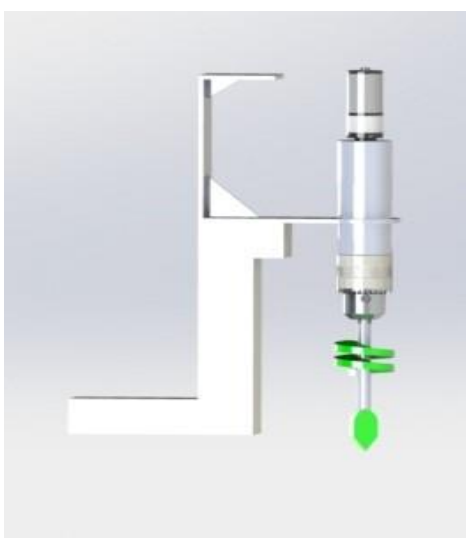

Figura. 5 Herramienta de perforación.

Los elementos (figura6) del dispensador se diseñaron en SolidWorks y se fabricaron mediante manufactura aditiva con material PLA.

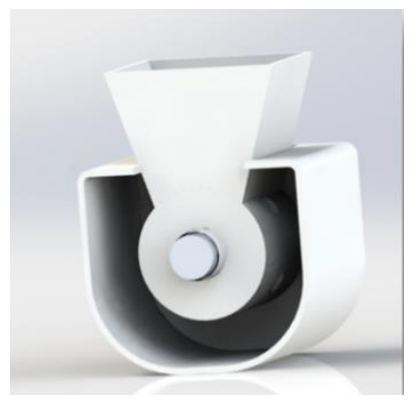

Figura. 6 Mecanismo dispensador de semillas.

\section{Subsistema de Energía:}

- Batería de Litio de $12 \mathrm{~V}$ : la capacidad de la batería es de 7500mah, su tamaño es el adecuado para el espacio en el armazón del robot.

- Regulador de Voltaje LM7805: se colocaron 4 reguladores de voltaje para evitar sobrecalentamiento en la placa y los componentes.

Subsistema de Comunicaciones:

- Para el sistema de procesamiento se tomaron dos microcontroladores: estos traducirán la información percibida por los sensores y recibirán los comandos del usuario. El dispositivo que va a procesar el radio control por medio del módulo de radiofrecuencia de $2.4 \mathrm{Ghz}$ es PIC18F45K22. También se conectará un segundo microcontrolador a la placa que es el ATMEGA328P (tabla1) para la señal de los sensores, y modulo GPS.

- Programación: se utilizará el programa de Arduino para realizar la programación necesaria 
para el proyecto, también se realizará un programa con Visual Studio Conde para la creación del SCADA.

Tabla 1. Características del ATMEGA328P

Subsistema de Geolocalización:

Modulo GPS: Se escogió el módulo GPS GY-NEO6MV2 descrita en la (tabla2) por su compatibilidad con el ATMEGA328P, con este el usuario conocerá la localización del robot dentro del terreno de plantación [7].

Tabla 2. Características del Módulo GPS

\begin{tabular}{|c|c|}
\hline $\begin{array}{c}\text { Modulo GPS GY- } \\
\text { NEO6MV2 }\end{array}$ & Valor \\
\hline $\begin{array}{l}\text { Rango de Suministro de } \\
\text { Energía }\end{array}$ & $3 V-5 V$ \\
\hline Antena & Cerámica \\
\hline Tamaño de Antena & $25 \times 25 \mathrm{~mm}$ \\
\hline Tamaño de Modulo & $25 \times 35 \mathrm{~mm}$ \\
\hline
\end{tabular}

Subsistema de Sensores y Control:

- Driver dfrobot dri0041: este driver sustituye al puente $\mathrm{H}$ que normalmente ya viene hecho en circuitos integrados. El driver es mucho más eficiente que un puente $\mathrm{H}$ normal ya que ya viene programado, este driver ayuda a que los motores puedan girar en ambos sentidos, avance $\mathrm{y}$ retroceso, ayudando al robot a tener una mejor movilidad [8].

- Higrómetro: se escogió el higrómetro FC-28 por su compatibilidad con el ATMEGA328P, enviara datos al usuario acerca de la humedad de la tierra en donde desea sembrar.[9]

- $\quad$ Sensor BME280: este sensor puede leer tres variables (temperatura, humedad y presión del aire) por lo cual es una gran ayuda al momento de fabricar la placa ya que requiere de menos espacio.[10]

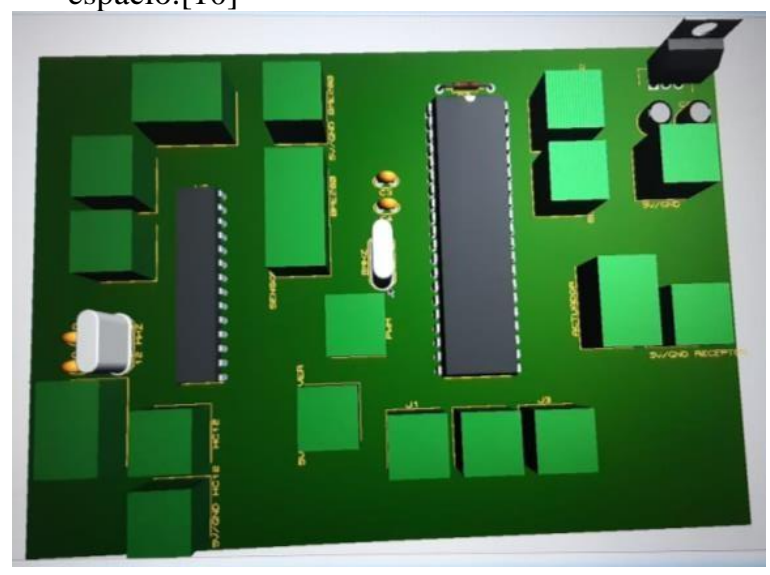

Figura. 7 Primer Diseño de la placa.

\section{IV: Integración de Partes}

Siguiendo los pasos de la metodología se realizaron las pruebas de los componentes que se utilizaron en los

\begin{tabular}{|l|l|}
\hline \multicolumn{1}{|c|}{$\begin{array}{c}\text { Nombre del } \\
\text { parámetro }\end{array}$} & \multicolumn{1}{c|}{ Valor } \\
\hline $\begin{array}{l}\text { Memoria de Programa } \\
\text { (KB) }\end{array}$ & 32 \\
\hline Timers & 2 de 8 bits y 1 de 16 bits \\
\hline Convertidores ADC & 5 \\
\hline Control PWM & $\mathrm{Si}$ \\
\hline Comparadores & 1 \\
\hline Oscilador Interno & $\begin{array}{l}8 \text { (MZ) usamos el de } \\
16(\mathrm{MZ}) \text { por que sube la } \\
\text { frecuencia del PWM }\end{array}$ \\
\hline Numero de Puertos & 23 \\
\hline
\end{tabular}

subsistemas.

1) Llantas

Mediante SolidWorks Express, se realizaron pruebas de estrés sobre las llantas, añadiendo cargas que determinaran el esfuerzo máximo permisible (figura8).

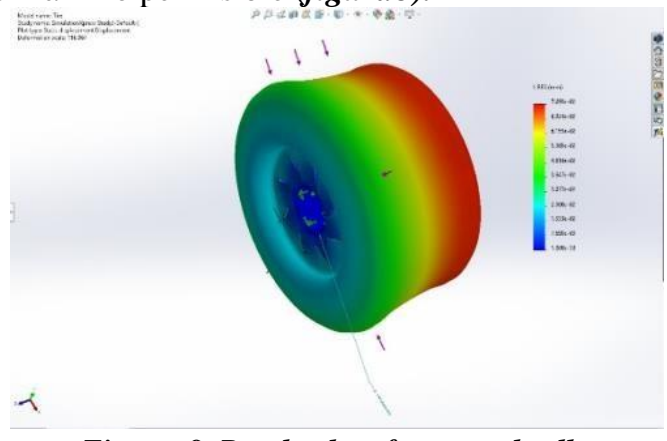

Figura. 8. Prueba de esfuerzo en las llantas.

2) Patas del ROVER

Se procedió a hacer un análisis de estrés a la pieza más comprometida que eran las patas del ROVER (figura9) para verificar que el material con el que fueron fabricadas, ABS, soportaran el peso del ROVER. Al hacer el análisis se visualizó deformación por exceso de carga, por lo que para confirmar los resultados se procedió a realizar un análisis de fatiga.

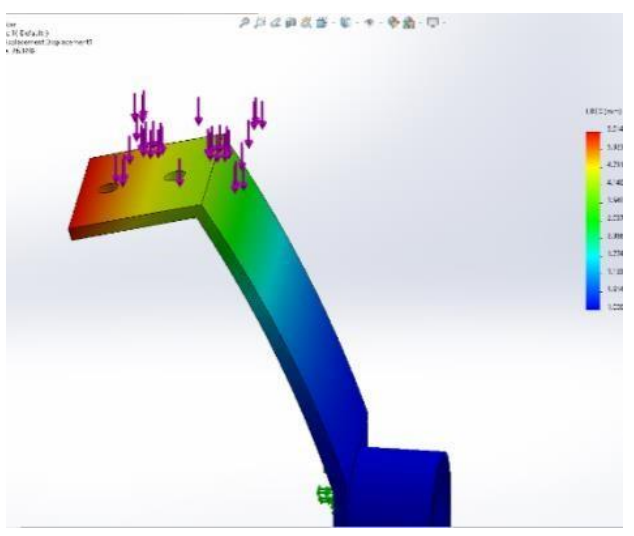

Figura.9 Análisis de Estrés.

En el análisis de fatiga se pudo confirmar que las patas del ROVER (figura10) estaban comprometidas y necesitaban

$1^{\text {th }}$ LACCEI International Multi-Conference for Engineering, Education, and Technology: "Global Partnerships for Development and Engineering Education”, 19-21 July 2017, Boca Raton Fl, United States. 
refuerzos debido a que los soportes cederían a las pocas horas de uso.

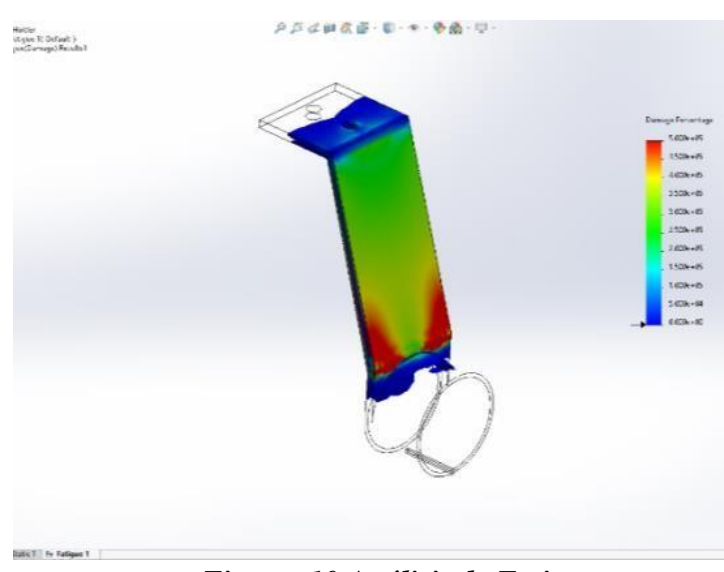

Figura. 10 Análisis de Fatiga.

Una técnica utilizada para el diagnóstico y supervisión de las piezas del ROVER fue el análisis de vibración de estas. Al observar la simulación (figura11) se concluyó que las deformaciones debido a vibraciones son nulas, esto se debe a que el motor usado es un motor DC y las únicas vibraciones experimentadas por la pieza se debían al movimiento del ROVER sobre el terreno, no obstante, las vibraciones eran mínimas y no repercutían daños sobre las piezas.

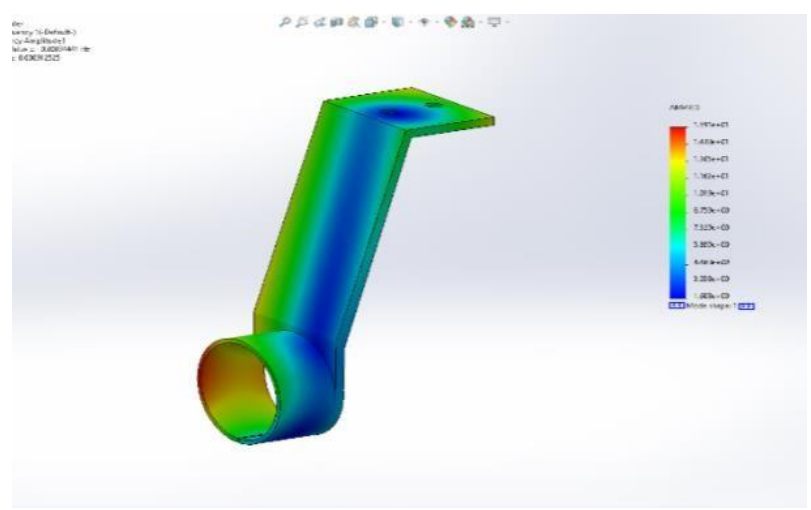

Figura. 11 Análisis de Vibración.

\section{3) Motor}

Los motores se conectaron a una caja de carga para determinar su funcionamiento y la carga que consumían en tiempos de operación. Este dato determinaría las propiedades de la batería.

\section{4) Baterías}

Se energizo la placa y los motores directamente a la batería para medir la autonomía que proporcionara la batería. Los resultados obtenidos fueron de 2 horas continúas operando.

5) Cargador

Se realizo una prueba a las baterías, para verificar el tiempo de carga al $100 \%$.

\section{6) Regulador de Voltaje}

Se conecto la batería a la placa electrónica que contiene los reguladores de voltaje para verificar la medición de voltaje obtenido.

\section{7) Microcontroladores}

Se elaboro una programación para realizar las pruebas en los microcontroladores y determinar el funcionamiento de los puertos PWM, de los sensores y de la transmisión de datos al sistema SCADA.

\section{8) SCADA}

Se procedió a observar que el SCADA (figura12) obtuviera correctamente los datos enviados por los microcontroladores y que los almacenara para un futuro análisis de estos.

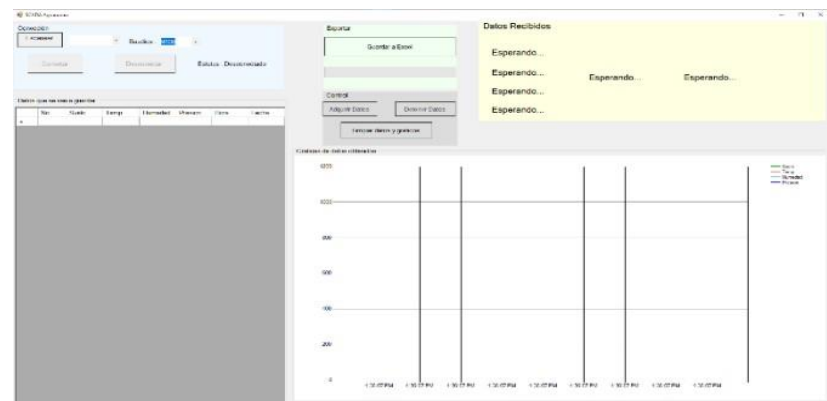

Figura. 12 SCADA desarrollado para el monitoreo de lecturas. 9) Programación

Se realizo un diagrama de flujo (figura13) que sirve como referencia para comprender la lógica de control y así mismo el proceso de tele operación del prototipo.

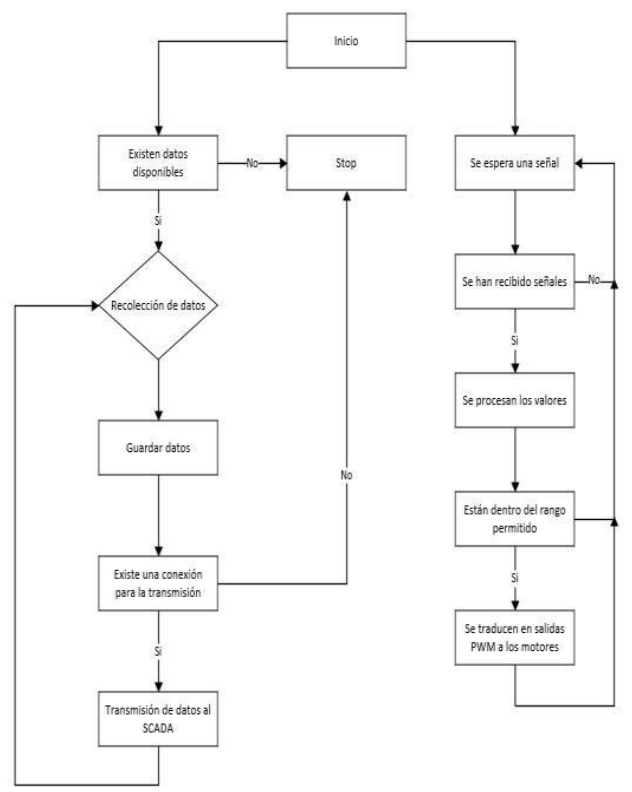

Figura. 13 Diagrama de Flujo.

\section{0) GPS}

Se energizo el módulo GPS para poder observar la precisión de las coordenadas que enviaba al usuario y poder calibrarlo correctamente para un óptimo funcionamiento.

\section{E. Etapa V: Integración de los subsistemas}

En la quinta etapa se integran las partes de los subsistemas para comprobar su comportamiento y funcionamiento en conjunto. 
Integración del Subsistema Mecánico:

Se utilizó SolidWorks Motion para realizar una simulación de movimiento y determinar la velocidad y torque (figura14) de los motores en desplazamiento.

Piezas de soporte: Se verificó que las piezas no sufrieran deformaciones plásticas o elásticas por la carga mecánica y se integró el efector final al rover para verificar que el peso del efector no desequilibrara la estructura del rover.

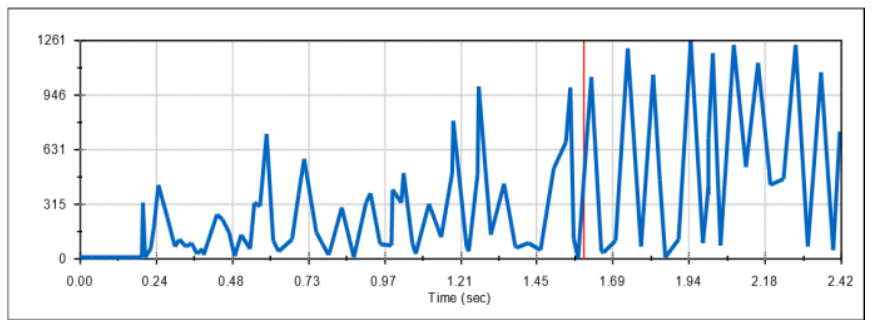

Figura. 14 Torque de los motores en superficie irregular.

1) Integración del Subsistema de Energía

Se conectaron las baterías a los motores y a la placa electrónica verificando las mediciones de voltaje a la entrada y salida de los reguladores.

\section{2) Integración del Subsistema de Comunicaciones}

Se cargó la programación a los microcontroladores para ver la interacción entre el programa y los microcontroladores y verificar que los datos censados coincidieran con los visualizados en el SCADA.

\section{3) Integración del Subsistema de Geolocalización}

Se incorporo el módulo GPS GYNEO6MV2 ya calibrado al Arduino, el cual proporcionaba una mejor recepción satelital.

4) Integración del Subsistema de Sensores y Control

Se incorporaron y se soldaron todos los sensores ya conectados con los microprocesadores a la placa electrónica y se realizaron las últimas pruebas de voltaje y funcionamiento de los sensores en conjunto con los microprocesadores.

\section{F. IV: Prototipo de laboratorio \\ 1) Desarrollo electrónico}

El desarrollo electrónico implicó la fabricación de dos placas electrónicas (figura15), presentando fallas de diseño en el primer modelo por sobrecalentamiento debido al uso de un único regulador de voltaje y al reducido tamaño de la placa, desproporcional a la cantidad de componentes. El segundo modelo constaba de 4 reguladores de voltajes y un mayor espaciamiento entre sus componentes lo que mitigo la emisión de calor.

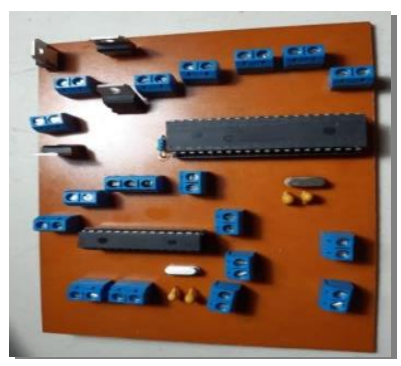

Figura. 15 Placa terminada.

2) Desarrollo estructural

El sistema mecánico se prueba por separado mediante simulaciones en SolidWorks Xpress, asimismo se realizan pruebas de campo en las que se evidencio la dificultad del Rover para sobrellevar su propio peso, presentado problemas de rigidez en los soportes de sus llantas y conflicto al momento de pivotear.

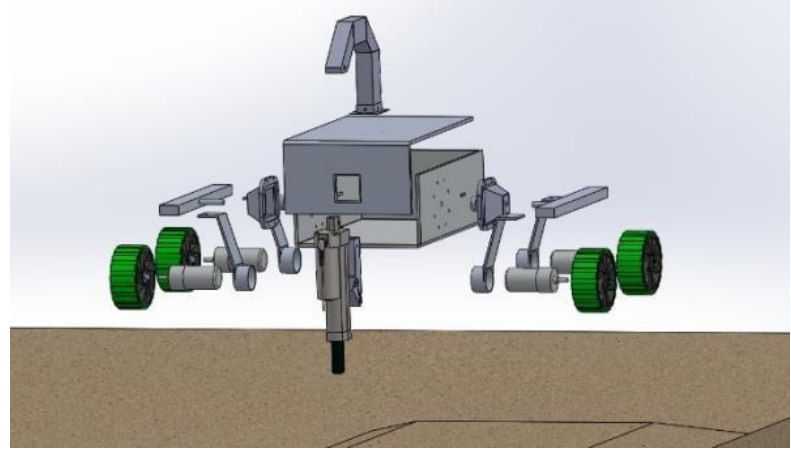

Figura. 16 Vista explosionada de toda la estructura.

3) Desarrollo de efector final

El efector final posee la capacidad de ser desmontable y adaptable a otros diseños de Rovers. El sistema consta de un mecanismo de perforación y un mecanismo dispensador de semillas. Con respecto al primero su diseño se basa en un concepto de taladro, de manera que su función es perforar la tierra por medio de una herramienta tipo broca cuyo movimiento giratorio remueve la tierra, abriendo un agujero de diámetro de $4 \mathrm{~cm}$, no obstante, este varía según la herramienta de perforación que se instale. Además, la herramienta alcanza una profundidad optima de $4 \mathrm{~cm}$ por debajo de la superficie de la tierra.

El mecanismo dispensador funciona mediante un concepto de tolva y rodillo, con accesorios intercambiables adecuándose al tipo de semilla.

Ambos sistemas son accionados y controlados de manera electrónica logrando automatizar sus funciones de acuerdo con las condiciones de la tarea a realizar en el proceso agrícola.

Se decidio por situar el efector final en la parte frontal del rover tal como se muestra en la (figura 17)formando una sola estructura entre el mecanismo de taladro y el dispensador de semillas de manera que su diseño sea mas compacto. 


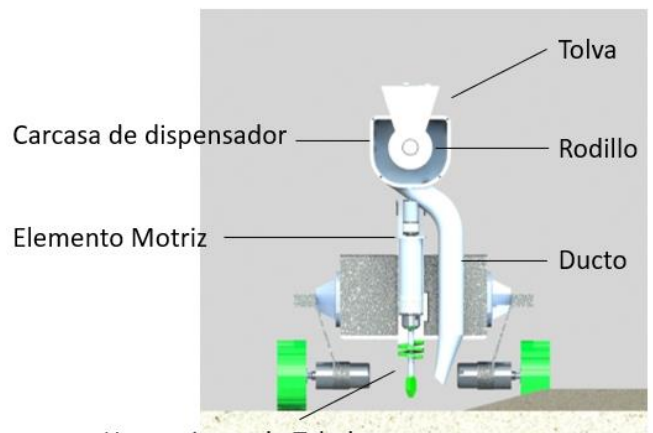

Herramienta de Taladro

Figura. 17 Ensamble Efector final y Rover.

\section{RESULTADOS FINALES}

Estructura del rover

Como se mencionó con anterioridad el Rover presento problemas de pivoteo, lo que llevo a implementar una rueda de giro sin fin, de un diámetro de $15 \mathrm{~mm}$ en la parte trasera y frontal de su estructura logrando equilibrar su sistema en los momentos de giro. Asimismo, en los soportes de sus llantas el Rover (figura 18) se reforzo para evitar las flexiones que se generaban debido a su peso.

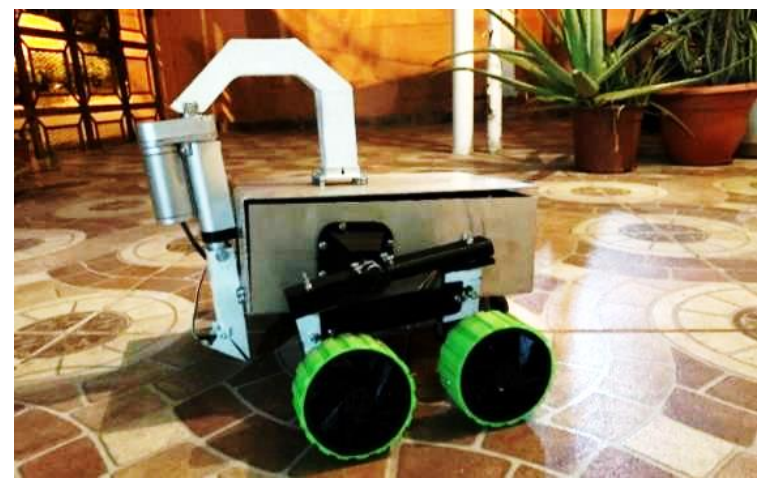

Figura. 18 Prototipo final.

\section{1) Muestreo de semillas}

Para las pruebas realizadas del efector final y para el diseño de la pieza "rodillo" del dispensador de semillas se tomó como referencia las semillas de frijoles, tomando una muestra para medirla con un instrumento de medición, el vernier o calibrador y concluyendo que el promedio y error estándar para las dimensiones de las semillas fueron: longitud: $1.112 \pm 0.0458 \mathrm{~cm}$, ancho: $0.579 \pm 0.033 \mathrm{~cm}$; grosor: $0.459 \pm 0.024 \mathrm{~cm}$.

2) Dispensador de semilla

El mecanismo dispensador de semillas consta de 5 elementos para su funcionamiento y estructuración: actuador, el cual será un motor paso a paso Nema 17 que ejerce un torque de $362 \mathrm{mN} / \mathrm{m}$ capaz de mover el eje sobre el cual ira dispuesto el rodillo; eje que ira acoplado al motor; rodillo de semillas con 5 perforaciones en donde se deposita la semilla y se traslada la a lo largo del movimiento giratorio hasta su salida; tolva encargada de mantener el dosificador abastecido; housing del mecanismo que encierre el rodillo y ducto semillero a través del cual cae la semilla por gravedad desde la tolva hasta el agujero.

3) El sistema de excavación de agujero por taladro

La profundidad de taladro se logró con la ayuda de un pistón eléctrico de $12 \mathrm{~V}$ con capacidad de estiramiento de $5 \mathrm{~cm}$, el pistón suspende la herramienta de taladro $1 \mathrm{~cm}$ sobre la superficie de la tierra, por lo tanto, la profundidad máxima de taladro es de $4 \mathrm{~cm}$ cumpliendo con la profundidad estándar para este tipo de semillas.

El sistema consistió en otros elementos importantes los cuales fueron adquiridos en el comercio y adaptados para el funcionamiento requerido. El husillo portabrocas motorizado, gira a 600rpm y su torque se ajusta manualmente a un máximo de $10 \mathrm{Nm}$, tiene una capacidad de ajuste de broca de $0.8-10 \mathrm{~mm}$. El taladro funciono como elemento motriz para la herramienta e hizo que la perforación de agujeros se realizara más fácil con la ayuda del pistón. El diseño de las aspas de la herramienta permitió que la tierra se removiera del agujero cuando el pistón se movía en su carrera de retorno.

\section{Herramienta de perforación}

Para definir la herramienta que se utilizó se procedió a calcular la fuerza mínima de empuje necesaria del pistón para perforar el suelo, tomando como referencia parámetros de resistencia a la compresión de los suelos tabla 3, obteniendo una fuerza mínima de empuje de $60 \mathrm{~N}$ para un suelo medio con $300 \mathrm{kN} / \mathrm{m}^{2}$ de resistencia. Tabla 3. Valores orientativos de resistencia a la compresión

\begin{tabular}{|c|c|}
\hline Tipo de suelo & $\mathbf{q u}\left(\mathbf{k N} / \mathbf{m}^{\mathbf{2}}\right)$ \\
\hline Suelos muy flojos o blandos & $0-80$ \\
\hline Suelos flojos o blandos & $80-150$ \\
\hline Suelos medios & $150-300$ \\
\hline Suelos compactos o duros & $300-500$ \\
\hline
\end{tabular}

$$
\text { Fuente:[12] }
$$

4) Pruebas y Validación de prototipo

En este apartado se especifican las pruebas experimentales de validación realizadas al prototipo para garantizar su funcionalidad al aire libre. Las pruebas fueron realizadas en dos espacios exteriores diferentes, terrenos planos con mínimas irregularidades debidos al diseño del rover sobre el cual se montaría la herramienta. Las tierras mostraban diferencias en su densidad, una era más compacta y la otra más suelta.

\section{Prueba en tierra cohesiva mecanismo}

$\mathrm{Al}$ realizar la primera prueba como se observa en la (figura 19) el trabajo simultaneo del pistón y el taladro facilito la perforación, el taladro respondió a las demandas de torsión 
auxiliando a la broca, sin embargo, las semillas en su mayoría caían fuera del agujero.

En cuanto al rover debido a la desproporción de carga. El mecanismo hacía que el rover se inclinara hacia enfrente y la vibración generaba mucho movimiento en el rover, esto intervenía en el funcionamiento del efector final. Así que se optó por agregar una rueda en la parte frontal del rover para estabilizarlo y evitar que influyera en las pruebas de validación del efector final.

La herramienta de perforación está prevista a que baje una profundidad de $4 \mathrm{~cm}$ y así mismo una vez terminada la operación suba la misma distancia quedando en la superficie del agujero para evitar rebotes entre la semilla y las aspas de la broca.

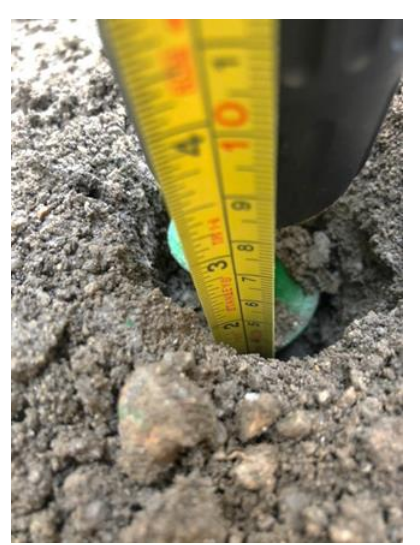

Figura. 19. Perforación sobre suelo cohesivo con profundidad de $4 \mathrm{~cm}$.

Prueba suelo granular húmedo

En la tierra granular mostrada en la (figura20) las pruebas mostraban leves diferencias en los resultados al ser la tierra más suelta el taladro removía con mayor facilidad. Sin embargo, las semillas continuaban rebotando sobre el aspa de la broca y por consiguiente no caía en el agujero.

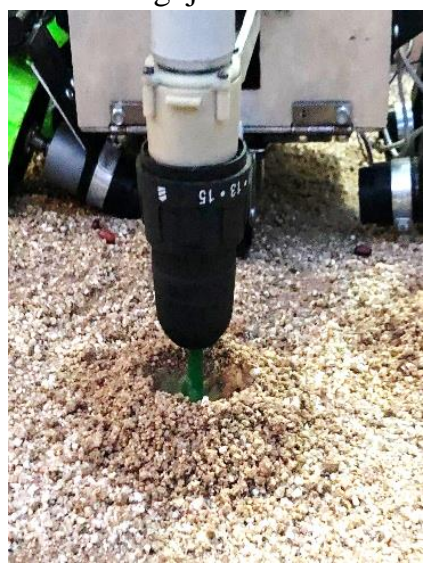

Figura. 20 Prueba sobre suelo granular

En todas las pruebas realizadas sobre terrenos planos la profundidad máxima de perforación fue de $4 \mathrm{~cm}$. El diseño del rover está previsto en superficies planas y consigo la herramienta de perforación. En teoría no debería de haber ninguna diferencia en la profundidad de los agujeros si se realizan sobre terrenos sin irregularidades, de lo contrario la inclinación del rover afectaría la eficiencia del efector final para perforar agujeros.

Para optimizar el funcionamiento del efector se aplicaron mejoras en el diseño de algunas de sus piezas. Se ajusto el largo de la herramienta, el distanciamiento entre las aspas y se reemplazó la punta triangular de la herramienta por una broca de acero de alta velocidad con un ángulo de penetración de 118 grados en su punta. Se modifico la boquilla del ducto dispensador inclinándola a un ángulo de 20.72 grados, logrando así que la semilla caiga directa en el agujero.

En un principio de realizaron 40 pruebas con el efector final sobre terreno llano de las cuales el porcentaje de precisión en la cantidad de semillas depositadas dentro del agujero se muestra en el siguiente gráfico (Figura21). Concluyendo que el margen de error se debía a que el exceso de tierra acumulada alrededor del agujero creaba una barrera entre la semilla y el agujero.

\section{Cantidad de semillas depositadas

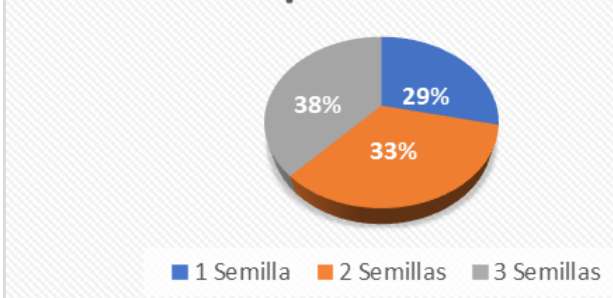

Figura. 21 Porcentaje de precisión del dispensador de semillas.

Para mejorar la precisión de semillas sembradas se añadieron fajillas plásticas entrelazadas en la broca, para barrer la tierra acumulada en el perímetro del agujero. Realizando 15 pruebas más se demostró lo siguiente (figura 22): el $87 \%$ de las veces equivalente a 13 dosis de semillas, todas las semillas cayeron dentro del agujero presentado diferencia en la cantidad ya sea de 1, 2 o 3 debido al tamaño de la semilla. Solo en dos dosis cayeron $2 / 3$ y $1 / 2$ respectivamente fuera del agujero. Aumentando la precisión en el dispensador.

\section{CANTIDAD DE SEMILLAS DEPOSITADAS}

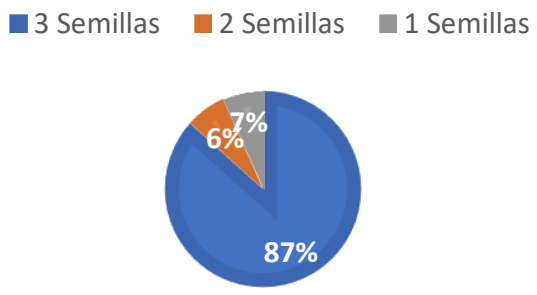

Figura 22 -Porcentaje de precisión del dispensador de semillas. 
El prototipo final tiene el siguiente funcionamiento, el efector final baja y perfora un agujero de $4 \mathrm{~cm}$ con la ayuda simultanea de un actuador lineal que ejerce una fuerza de $60 \mathrm{~N} \mathrm{y}$ un taladro girando a 600 rpm y con un torque de $10 \mathrm{Nm}$. Alcanzado el máximo estiramiento del pistón y su retorno se procede a accionar el motor del mecanismo dispensador, el cual se encuentra con la tolva abastecida. El motor impulsa el giro del rodillo, a través del cual las semillas se movilizan hacia el compartimento externo de la tolva y caen por gravedad a la carcasa del dispensador a través de un agujero que conduce hacia un ducto reductor por donde la semilla desliza cayendo hasta el agujero.

\section{CONCLUSION}

Las pruebas concernientes al prototipo comprobaron su funcionalidad manteniendo un control constante y preciso en la orientación de los motores, así como efectividad en la adquisición de datos en el periodo de tiempo funcional de los sensores. Se verifico la correcta comunicación inalámbrica de la estación de monitoreo SCADA, así como el control optimo del prototipo en una distancia de 300 metros. Llevando a cabo las pruebas de esfuerzo realizadas en las partes mecánicas del rover, se confirmó la dureza de estas y su viabilidad en un periodo a largo plazo. El rover cumple con su objetivo principal de sembrar de manera precisa e individualizada un máximo de 3 semillas de frijol a una profundidad de $4 \mathrm{~cm}$ bajo la superficie de la tierra sobre terrenos planos, esto a través de su efector final diseñado para siembra operaciones de siembra.

\section{DISCUSION}

El desarrollo de un dispositivo de medición controlado electrónicamente da la posibilidad de reducir el tamaño total del aparato. El dispensador de semillas tiene el potencial de reducir su tamaño total en gran medida con la incorporación de accionamiento electrónico en el sistema. En los equipos dispensadores existentes de tipo discos como los dispensadores al vacío, existe un factor limitante importante y es el tamaño del disco de semillas requerido para lograr el proceso de individualización. Estos discos de vacío funcionan en cooperación con la presión negativa del aire para recoger y colocar semillas individuales en el tubo de semillas. La incorporación de un componente tan grande en el diseño limita la colocación del medidor de semillas a áreas cercanas al suelo suelo [13]-[15].

A diferencia de lo anteriormente mencionado uno de los mayores esfuerzos en el desarrollo del rover fue lograr un diseño compacto de su efector final logrando que a través de medios electrónicos y un diseño ergonómico su disposición no estuviera sujeta a una única estructura y que las técnicas de siembra se dieran a través de medios precisos e individualizados. Tal como [16] describe un diseño de efector final de husillo motorizado, se concluyó que para penetrar la tierra y formar un agujero con diámetro delimitado sería factible y favorecedor un efector basado en este concepto en donde la herramienta de perforado penetre impulsado por un giro rotacional y una fuerza aplicada a la misma, asimismo que permita intercambiar entre herramientas adaptándose al tipo de semillas y su requerimiento necesario en diámetro de perforación para que germine en un espacio adecuado.

\section{REFERENCIAS}

[1] Machado, N., Rossato, V., Coronel, D., \& Schuh, Da. (2018). El desempeño de la agricultura en américa latina: Análisis de la eficiencia y eficacia de la región. 26(33). http://dx.doi.org/10.18359/rfce.2775

[2] Fassa, A., Fiori, N., Meucci, R., Faria, N., \& de Carvalho, M. (2020). Dolor cervical entre agricultores que producen tabaco en el sur de Brasil. https://doi.org/10.18294/sc.2020.2307

[3] Magaña, A., Lopez, A., \& Gutierrez, V. (2019). Situación social y tecnológica en el manejo del agua para riego en Puebla, México. 29. https://doi.org/10.15174/au.2019.2114.

[4] Garrido-Rubiano, M., Martínez-Medrano, J., MartínezBautista, H., \& Granados-Carvajal, R. (2016). Pequeños productores de maíz en el Caribe colombiano: Estudio de

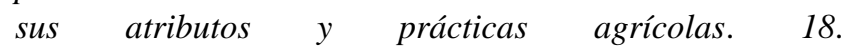
http://dx.doi.org/10.21930/rcta.vol18_num1_art:556

[5] Solaque, L., Sanchez, G., \& Riveros, A. (2019). Control de velocidad traslacional y orientación de un robot dedicado a agricultura de precisión. 15, 2-3. https://doi.org/10.33571

[6] Yu, J., Zhang, J., Wu, N., Zheng, C., \& Zhang, D. (2019). Coding Style of

C Program from the Perspective of Software Engineering. IOP Conference Series: Materials Science and Engineering, 563, $\quad 052073 . \quad$ https://doi.org/10.1088/1757899X/563/5/052073

[7] uBlox "Datasheet of GPS GY -NEO6MV 2" s.f https://www.ublox.com/sites/default/files/products/docume nts/NEO-

6_DataSheet_(GPS.G6-HW-09005).pdf

[8] Dfrobot "Datasheet of DRI004" s.f. https://wiki.dfrobot.com/7A_Dual_DC_Motor_Driver_SK U__DRI0041

[9] Bosch "Densortec "Datasheet of BME280"

s.f.https://www.digchip.com/datasheets/8773796-bme280board-mounthumidity.html

[1] J. P. Vasconez, G. A. Kantor, and F. A. Auat Cheein, "Human-robot interaction in agriculture: A survey and current challenges," Biosystems Engineering, vol. 179, pp. 35-48, Mar. 2019, doi: 10.1016/j.biosystemseng.2018.12.005.

[2] L. Yu, H. Qin, and P. Xiang, "Incentive mechanism of different agricultural models to agricultural technology information management system," Sustainable Computing: Informatics and Systems, vol. 28, p. 100423, Dec. 2020, doi: 10.1016/j.suscom.2020.100423.

[3] Z. Zhai, J. F. Martínez, V. Beltran, and N. L. Martínez, "Decision support systems for agriculture 4.0: Survey and 
challenges," Computers and Electronics in Agriculture, vol. 170, p. 105256, Mar. 2020, doi: 10.1016/j.compag.2020.105256.

[4] A. Bechar and C. Vigneault, "Agricultural robots for field operations. Part 2: Operations and systems," Biosystems Engineering, vol. 153, pp. 110-128, Jan. 2017, doi: 10.1016/j.biosystemseng.2016.11.004.

[5] A. Sharda, A. Franzen, D. E. Clay, and J. D. Luck, "Precision Variable Equipment," in ASA, CSSA, and SSSA Books, D. Kent Shannon, D. E. Clay, and N. R. Kitchen, Eds. Madison, WI, USA: American Society of Agronomy and Soil Science Society of America, 2018, pp. 155-168. doi: 10.2134/precisionagbasics.2016.0094.

[6] I. Charania and X. Li, "Smart farming: Agriculture's shift from a labor intensive to technology native industry," Internet of Things, vol. 9, p. 100142, Mar. 2020, doi: 10.1016/j.iot.2019.100142.

[7] J. Dario Enamorado, J. Luis Ordoñez Avila, M. Gustavo Ordoñez Avila, and O. Aguiluz Guevara, "Iot-SPA Billing System to Improve the Coffee Recollection in Beneficio Rio Frio, Honduras, Santa B\&\#xc1;Rbara," in 2020 The 4th International Conference on E-Business and Internet, New York, NY, USA, Oct. 2020, pp. 26-31. doi: 10.1145/3436209.3436382.

[8] J. Azeta, C. A. Bolu, F. Alele, E. O. Daranijo, P. Onyeubani, and A. A. Abioye, "Application of Mechatronics in Agriculture: A review," J. Phys.: Conf. Ser., vol. 1378, p. 032006, Dec. 2019, doi: 10.1088/17426596/1378/3/032006.

[9] J. Luis Ordoñez Avila, H. Fernando Jimenez, and A. Max Carrasco, "Spiral Cycle Implementation for Designing an All-Terrain Teleoperated Robot in Honduras," in 2020 6th International Conference on Robotics and Artificial Intelligence, New York, NY, USA, Nov. 2020, pp. 253257. doi: $10.1145 / 3449301.3449344$.

[10] J. L. O. Avila et al., "Study Case: Teleoperated Voice Picking Robots prototype as a logistic solution in Honduras," in 2020 5th International Conference on Control and Robotics Engineering (ICCRE), Apr. 2020, pp. 19-24. doi: 10.1109/ICCRE49379.2020.9096483.

[11] C. A. Morar, I. A. Doroftei, I. Doroftei, and M. G. Hagan, "Robotic applications on agricultural industry. A review," IOP Conf. Ser.: Mater. Sci. Eng., vol. 997, p. 012081, Dec. 2020, doi: 10.1088/1757-899X/997/1/012081.

[12] L. Juiña,Arellano,Llumipanta Roberto,Paul, "Diseño y construcción de una máquina perforadora de suelo para anclajes de diámetro 32 × $12000 \mathrm{~mm}$ de largo para la estabilización de taludes.” Jun. 2015. [Online]. Available: DOI: 10.17163/ings.n13.2015.03

[13] O. Arteaga et al., "Automation of a seed on tray seeder machine," IOP Conf. Ser.: Mater. Sci. Eng., vol. 872, p. 012003, Jun. 2020, doi: 10.1088/1757899X/872/1/012003.

[14] H. E. Ospina-Bayona, C. C. Sánchez-Torres, R. A. GarcíaLeón, H. A. Ballesteros-Ruiz, and B. C. Pérez-Lozano, "Design of a mechanical seeder," J. Phys.: Conf. Ser., vol.
1388, p. 012004, Nov. 2019, doi: 10.1088/17426596/1388/1/012004.

[15] S. Dutta, U. Shanker, S. Katiyar, V. Singh, M. N. Zafar, and J. C. Mohanta, "Development and Fabrication of an Autonomous Seed Sowing Robot," Materials Science and Engineering, p. 9, 2019.

[16] Y. Xie, W. Zou, Y. Yang, and J. Lia, "Design of robotic end-effector for milling force control," IOP Conf. Ser.: Mater. Sci. Eng., vol. 423, p. 012032, Nov. 2018, doi: 10.1088/1757-899X/423/1/012032. 